Ferrata Storti Foundation

\title{
Allogeneic peripheral blood stem cell transplantation with anti-thymocyte globulin versus allogeneic bone marrow transplantation without anti-thymocyte globulin
}

Frédéric Baron, ${ }^{1}$ Jacques-Emmanuel Galimard, ${ }^{2-5}$ Myriam Labopin, ${ }^{2-5}$ Ibrahim Yakoub-Agha, ${ }^{6}$ Riitta Niittyvuopio, ${ }^{7}$ Nicolaus Kröger, ${ }^{8}$ Laimonas Griskevicius, ${ }^{9}$ Depei Wu, ${ }^{10}$ Edouard Forcade, ${ }^{11}$ Carlos Richard, ${ }^{12}$ Mahmoud Aljurf, ${ }^{13}$ Grzegorz Helbig, ${ }^{14}$ Hélène Labussière-Wallet, ${ }^{15}$ Mohamad Mohty ${ }^{2-5}$ and Arnon Nagler ${ }^{2,16}$

Haematologica 2020

Volume 105(4):1138-1146

${ }^{1}$ Laboratory of Hematology, GIGA-I3, University of Liège and CHU of Liège, Liege, Belgium; ${ }^{2}$ EBMT Paris study office/CEREST-TC, Paris, France; ${ }^{3}$ Department of Haematology, Saint Antoine Hospital, Paris, France; ${ }^{4}$ Sorbonne University, Centre De Recherche Saint Antoine, INSERM UMR938, Paris, France; ${ }^{5}$ Sorbonne University, Paris,

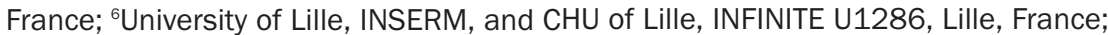
${ }^{7} \mathrm{HUCH}$ Comprehensive Cancer Center, Stem Cell Transplantation Unit, Helsinki, Finland; ${ }^{8}$ University Hospital Eppendorf, Bone Marrow Transplantation Center, Hamburg, Germany; 'Institute of Clinical Medicine, Vilnius University and Vilnius University Hospital Santaros Klinikos, Vilnius, Lithuania; ${ }^{10}$ First Affiliated Hospital of Soochow University, Department of Hematology, Suzhou, China; ${ }^{11} \mathrm{CHU}$ Bordeaux Hôpital HautLeveque, Pessac, France; ${ }^{12}$ Hospital U. Marqués de Valdecilla, Servicio de HematologíaIDIVAL, Santander, Spain; ${ }^{13}$ King Faisal Specialist Hospital \& Research Centre Oncology, Riyadh, Saudi Arabia; ${ }^{14}$ Silesian Medical Academy, University Department of Haematology and Bone Marrow Transplantation, Katowice, Poland; ${ }^{15}$ Centre Hospitalier Lyon Sud, Service Hematologie, Lyon, France and ${ }^{16}$ Division of Hematology and Bone Marrow Transplantation, The Chaim Sheba Medical Center, Tel-Hashomer, Ramat-Gan, Israel

\section{ABSTRACT}

\section{Correspondence:}

FRÉDÉRIC BARON

f.baron@ulg.ac.be

Received: May 28, 2019.

Accepted: August 13, 2019.

Pre-published: August 14, 2019.

doi:10.3324/haematol.2019.227603

Check the online version for the most updated information on this article, online supplements, and information on authorship \& disclosures: www.haematologica.org/content/105/4/1138

(C)2020 Ferrata Storti Foundation

Material published in Haematologica is covered by copyright. All rights are reserved to the Ferrata Storti Foundation. Use of published material is allowed under the following terms and conditions:

https://creativecommons.org/licenses/by-nc/4.0/legalcode. Copies of published material are allowed for personal or internal use. Sharing published material for non-commercial purposes is subject to the following conditions:

https://creativecommons.org/licenses/by-nc/4.0/legalcode, sect. 3. Reproducing and sharing published material for commercial purposes is not allowed without permission in writing from the publisher. relapse-free survival and other transplantation outcomes of acute myeloid leukemia (AML) patients given bone marrow (BM) without anti-thymocyte globulin (ATG) versus peripheral blood stem cells (PBSC) with ATG after myeloablative conditioning. In the cohort of patients receiving grafts from a human leukocyte antigen (HLA)-matched sibling donor, patients given PBSC with ATG $(n=1,021)$ and those given BM without ATG $(n=1,633)$ presented comparable severe GvHD-free relapsefree survival (GRSF)(hazard ratio [HR] $=0.9,95 \%$ confidence interval [CI]: $0.8-1.1, P=0.5)$ and overall survival ( $\mathrm{HR}=1.0,95 \% \mathrm{CI}$ : $0.8-1.2, P=0.8)$. They had however, a lower incidence of chronic GvHD (cGvHD) ( $\mathrm{HR}=0.7,95 \%$ CI: 0.6-0.9, $P=0.01$ ). In the cohort of patients receiving grafts from HLAmatched unrelated donor, patients given PBSC with ATG $(n=2,318)$ had better severe GvHD-free and relapse-free survival (GRFS) than those given BM without ATG $(n=303)(\mathrm{HR}=0.8,95 \% \mathrm{CI}: 0.6-0.9, P=0.001)$. They also had a lower incidence of $\mathrm{cGvHD}(\mathrm{HR}=0.6,95 \% \mathrm{CI}: 0.5-0.8, P=0.0006)$ and better overall survival ( $\mathrm{HR}=0.8,95 \% \mathrm{CI}$ : 0.6-1.0, $P=0.04$ ). In summary, these data suggest that PBSC with ATG results in comparable (in the case of sibling donor) or significantly better (in the case of unrelated donor) severe GRFS than BM without ATG in patients with AML in complete remission receiving grafts after myeloablative conditioning.

\section{Introduction}

A number of phase III trials have compared peripheral blood stem cells (PBSC) with bone marrow (BM) as the stem cell source for allogeneic hematopoietic cell transplantation in patients given grafts after myeloablative regimens. ${ }^{1,2} \mathrm{~A}$ metaanalysis of trials performed in patients given grafts from human leukocyte antigen (HLA)-matched sibling donors (MSD) revealed that the use PBSC of instead of BM was associated with a higher incidence of grade III-IV acute graft-versus-host dis- 
ease (aGvHD) and (extensive) cGvHD, as well as a lower incidence of relapse (IR) and better overall survival (OS) in the subgroup of patients with late-stage disease. ${ }^{3}$ Further, the only phase III trial performed in patients given grafts from HLA-matched unrelated donors (MUD) showed that patients randomized to the BM group had a lower inci-
A

OS in MSD

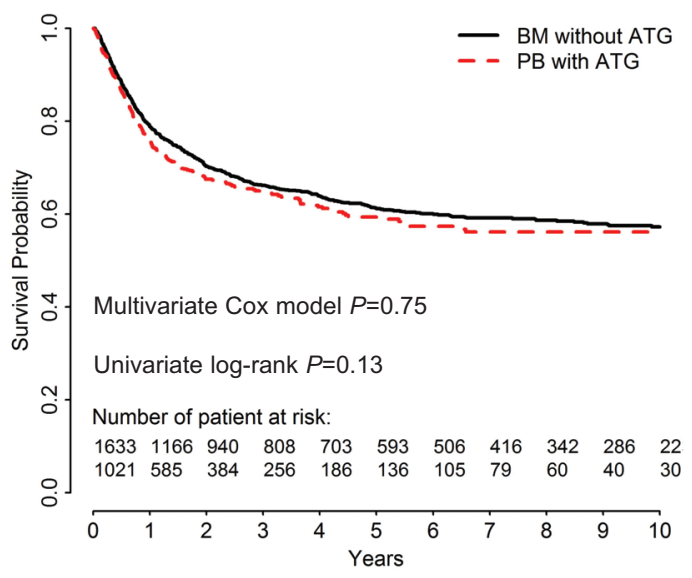

C

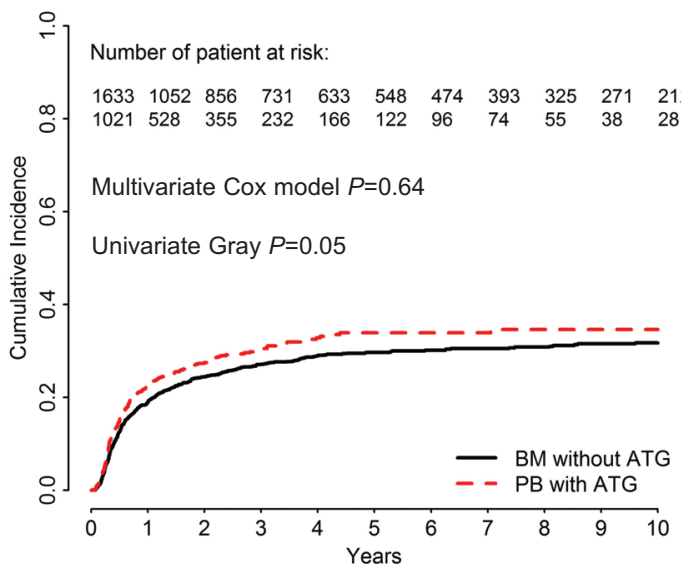

E

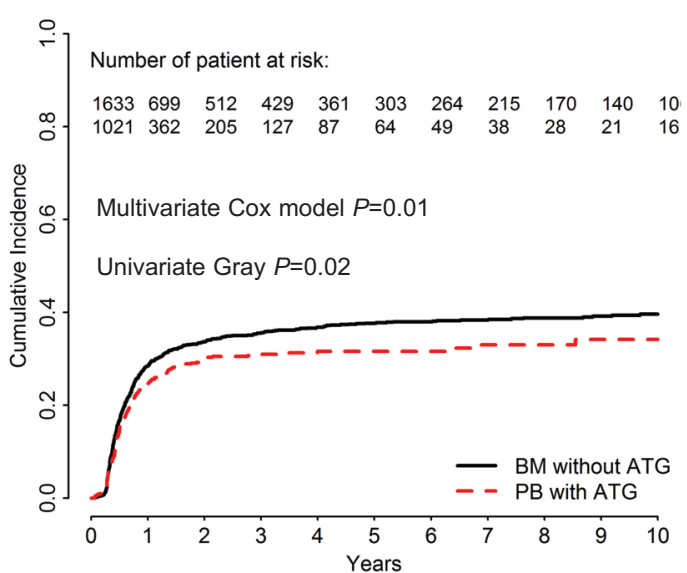

B

LFS in MSD

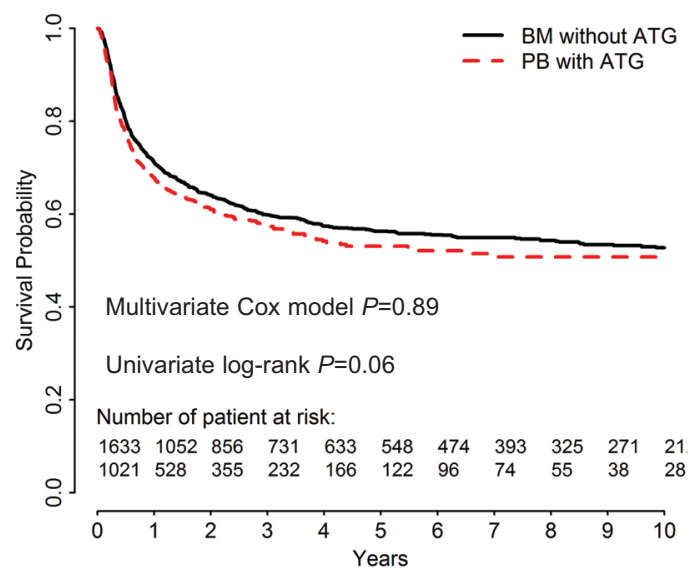

NRM in MSD

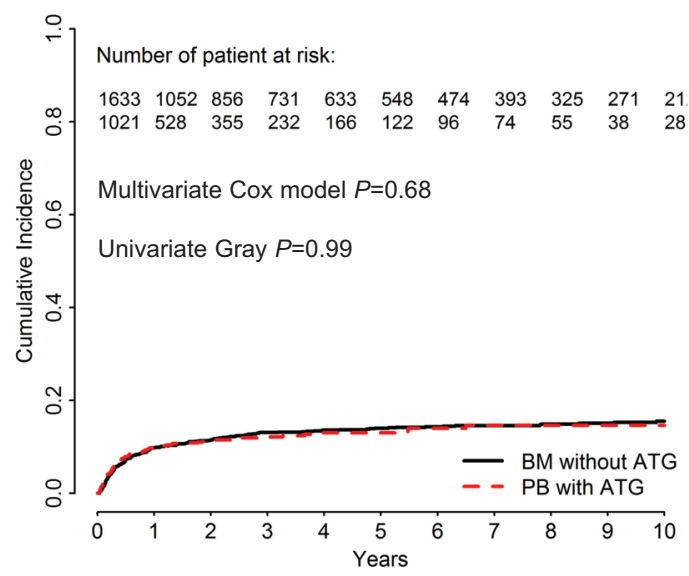

F

GRFS in MSD

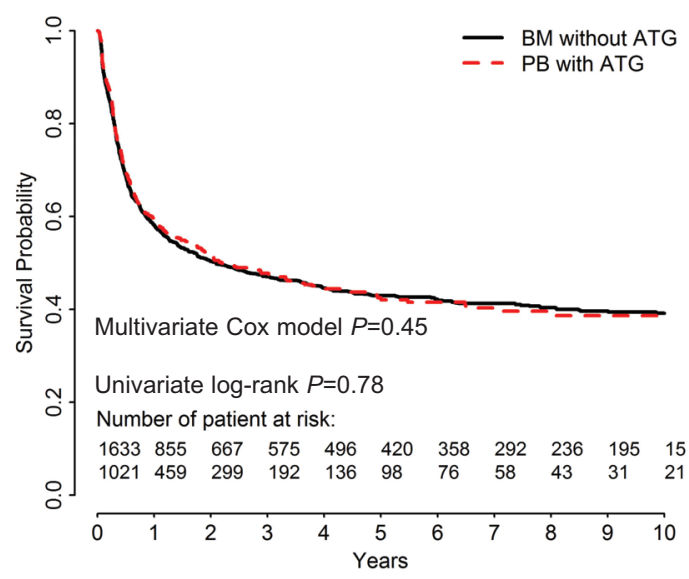

Figure 1. Comparison of outcomes in bone marrow (BM) patients without anti-thymocyte globulin (ATG) and in those given peripheral blood (PB) stem cells with ATG (the dotted line shows the PBSC with ATG curve adjusted for relevant covariates) in the cohort of patients receiving grafts from HLA-identical sibling donor (MSD). (A) Overall survival (OS), $P=0.13$. (B) Leukemia-free survival (LFS), $P=0.065$. (C) Incidence of relapse (RI), $P=0.0496$. (D) Non-relapse mortality (NRM), $P=0.989$. (E) chronic graft-versus-host disease (cGvHD), $P=0.0186$. (F) GvHD-free and relapse-free survival (GRFS), $P=0.782$. 
dence of $\mathrm{cGvHD}$, similar OS, and better patient-reported outcomes at 5 years after transplantation than those randomized to the PBSC arm. ${ }^{4,5}$ This might suggest that, at least in the MUD setting, BM may be preferred over PBSC as a stem cell source in patients receiving grafts after myeloablative regimens. In concordance with these findings, a recent European Group for Blood and Marrow Transplantation (EBMT) study observed a better GvHDfree relapse-free survival (GRFS) with BM than with peripheral blood $(\mathrm{PB})$ as the stem cell source. ${ }^{6}$

In the last two decades, five phase III trials have assessed the impact of anti-thymocyte globulins (ATG) on transplantation outcomes in patients given grafts (mainly PBSC) from MSD $(n=1)^{7}$ or MUD $(n=4)^{8-11}$ These five trials demonstrated a lower incidence of cGvHD with ATG. ${ }^{12}$ Two of three trials assessing the impact of ATG on GRFS' ${ }^{13}$ observed better GRFS in patients randomized to ATG ${ }^{7,10}$ while the study by Soiffer et al. failed to demonstrate this effect. ${ }^{11}$ ATG was also found to improve GRFS in a registry study including data from patients given grafts from HLA-identical siblings after myeloablative fludarabine + busulfan regimens. ${ }^{13}$

These findings prompted us to compare transplantation outcomes in AML patients following BMT without ATG versus $\mathrm{PBSC}$ transplantation with ATG in the first or second complete remission (CR).

\section{Methods}

\section{Ethics}

The scientific board of the Acute Leukaemia Working Party (ALWP) of the European Society for Blood and Marrow Transplantation (EBMT) approved this research project. The study was conducted according to the Declaration of Helsinki and Good Clinical Practice guidelines.

\section{Inclusion/exclusion criteria and study endpoints}

Inclusion criteria included adult patients, de novo or secondary AML, myeloablative conditioning, MSD or 10/10 HLA-matched unrelated donors (MUD), first or second CR, first allo-transplantation from 2000 to 2017 at an EBMT-affiliated center, and either BM without ATG, or PBSC with ATG. Exclusion criteria consisted of in vitro T-cell depletion of the graft, and prior administration of alemtuzumab. The primary endpoint was GRFS. Secondary endpoints included grade II-IV GvHD, RI, nonrelapse mortality (NRM), leukemia-free survival (LFS) and OS.

\section{Statistical analyses}

Myeloablative conditioning was defined as use of $\geq 6$ Gy TBI, busulfan $>8 \mathrm{mg} / \mathrm{kg}$, or melphalan $>140 \mathrm{mg} / \mathrm{m}^{2}$ as previously reported. ${ }^{14}$ The cytogenetic AML risk was determined as previously reported. ${ }^{15,16}$

NRM was defined as death without prior or current disease recurrence. Events in the composite endpoint GRFS included grade III-IV aGvHD, extensive cGvHD, relapse or death as previously reported. ${ }^{17}$

OS, LFS and GRFS were estimated using the Kaplan-Meier estimates. Cumulative incidence functions (CIF) were used for $\mathrm{RI}$ and NRM in a competing risk setting, since death and relapse are competing together. In estimating the cumulative incidence of aGvHD and cGvHD, we considered relapse and death to be competing events. Univariate analyses were done using Gray's test for CIF and the log-rank test for GRFS, OS and LFS. Since there were some imbalances between the groups we performed multivariate Cox models to further determine the impact of the graft type on transplantation outcome. These Cox models were adjusted for disease status at transplantation, female donor to male recipient or not, CMV serostatus, use or not of total body irradiation (TBI), year of transplantation, age at transplantation, primary versus secondary AML, and cytogenetic risk. In order to take into account the center effect, we introduced a frailty for each center into the model, as previously reported. ${ }^{18,19}$ Specifically, we introduced a random effect (also named frailty effect) in the Cox multivariate models. Then, the same random effect was shared by all patients within the same center. In order to assess whether there was a statistical interaction between the year of transplantation and the association between BMT without ATG versus PBSC with ATG and the incidence of relapse, we introduced the interaction term between cell source and year as a binary variable (with the median year by group as the cut-off) in the Cox multivariate model for relapse. Results are presented as hazard ratios (HR) and 95\% confidence intervals (95\% CI). The HR for year of transplantation corresponded to a 5 -year increase and to a 10-year increase for the age at transplantation. As planned in the synopsis, the analyses were done separately for MSD and MUD. The interaction between graft type (i.e. BM without ATG or PBSC with ATG) and donor type (MSD versus MUD) was confirmed for several outcomes (data not shown).

\section{Results}

\section{BM without ATG versus PBSC with ATG in MSD recipients}

\section{Patients}

A total of 2,654 MSD recipients met the inclusion/exclusion criteria, comprising 1,633 BM without ATG patients and 1,021 PBSC with ATG patients (Table 1). In comparison to BM without ATG recipients, PBSC with ATG patients were older (median 48 years vs. 40 years old), were transplanted more recently (median year of transplantation 2014 vs. 2008), less likely to have a good cytogenetic risk (10\% vs. $16 \%)$, more likely to have secondary AML $(11 \%$ vs. $6 \%)$, less frequently received TBI in the conditioning regimen $(12 \%$ vs. $32 \%)$, and were more frequently male patients receiving grafts from female donors ( $25 \%$ vs. $22 \%$ ).

\section{GvHD}

The 100-day incidence of grade $2-4$ aGvHD was $27 \%$ (95\% CI: $25-29 \%$ ) in BM without ATG recipients versus $18 \%$ (95\% CI: $16-20 \%)$ in PBSC with ATG patients $(P<0.001)$. For grades III-IV, the figures were $9 \%(95 \%$ CI: $8-11 \%)$ and $6 \%(95 \%$ CI: $4-7 \%)$, respectively $(P=0.002)$. In multivariate analyses, the difference for grade II-IV aGvHD between PBSC with ATG versus BM without ATG was no longer statistically significant ( $\mathrm{HR}=0.8,95 \%$ CI: 0.7-1.1, $P=0.12$ ) (Table 2) while the use of TBI in the conditioning was associated with a higher incidence of grade II-IV aGvHD (HR=1.4, 95\% C1: 1.1$1.8, P=0.004)$. In contrast, a more recent transplantation year was associated with a lower incidence of grade II-IV aGvHD (HR=0.8, 95\% CI: 0.7-0.9, $P=0.0004)$.

At 2 years the CI of cGvHD was $34 \%$ (95\% CI: $31-$ $36 \%$ ) in BM without ATG recipients versus $30 \%$ (95\% CI: $27-33 \%)$ in PBSC with ATG patients $(P=0.02)$ (Figure $1 \mathrm{E})$. This association remained statistically significant on multivariate analysis with a lower risk of cGvHD among PBSC with ATG recipients ( $\mathrm{HR}=0.7,95 \%$ CI: 0.6-0.9, 
$P=0.01)$. In contrast, female donor to male recipient $(\mathrm{HR}=1.5,95 \% \mathrm{C} 1: 1.3-1.7, P<0.001)$, and older age at transplantation (HR=1.07, 95\% C1: 1.0-1.14, $P=0.049)$ were associated with a higher incidence of $\mathrm{cGvHD}$.

\section{Relapse and non-relapse mortality}

The 2-year CI of relapse was 25\% (95\% CI: $22-27 \%$ ) in BM without ATG recipients versus $28 \%$ (95\% CI: $25-31 \%$ ) in PBSC with ATG patients $(P=0.0496)$ (Figure 1C). After

Table 1. Patient characteristics among human leucocyte antigen-identical sibling donor (MSD) recipients

\begin{tabular}{|c|c|c|c|}
\hline Variable & $\begin{array}{c}\text { BM without ATG } \\
(n=1633)\end{array}$ & $\begin{array}{c}\text { PBSC with ATG } \\
(n=1021)\end{array}$ & $P$ \\
\hline Follow up, median $(95 \% \mathrm{CI})$ & $6.1(5.9-6.5)$ & $2.3(2.1-2.5)$ & \\
\hline Age of patient at HSCT (years), Median (IQR) & $40(30-48)$ & $48(38-56)$ & $<0.0001$ \\
\hline $\begin{array}{l}\text { Sex, n (\%) } \\
\text { Male } \\
\text { Female } \\
\text { Missing }\end{array}$ & $\begin{array}{c}794(49) \\
838(51) \\
1\end{array}$ & $\begin{array}{c}535(53) \\
480(47) \\
6\end{array}$ & 0.04 \\
\hline \multicolumn{4}{|l|}{ Karnofsky, n (\%) } \\
\hline$<80$ & $30(2)$ & $29(3)$ & 0.33 \\
\hline$\geq 80$ & $1270(98)$ & $950(97)$ & \\
\hline Missing & 333 & 42 & \\
\hline
\end{tabular}

Status at transplant, $\mathrm{n}(\%)$

\begin{tabular}{|c|c|}
\hline CR1 & $1407(86)$ \\
\hline
\end{tabular}

CR2

$226(14)$

$128(13)$

Cytogenetic risk

$<0.0001$

\begin{tabular}{lcc} 
Good $^{1}$ & $190(16)$ & \\
Intermediate $^{2}$ & $804(70)$ & $428(20$ \\
Poor $^{3}$ & $158(14)$ & $136(381$ \\
Missing & 481 \\
\hline
\end{tabular}

AML type

Primary

Secondary

$1537(94)$

$96(6)$

$62(10)$

428 (68)

136 (22)

Year of transplant, Median (IQR)

2008 (2004-2012)

395

Sex mismatch, $n$ (\%)

Female to male

355 (22)

CMV donor/patient, n (\%)

$356(24)$

$908(89)$

$113(11)$

\begin{tabular}{lcc}
$-/-$ & $356(24)$ & $187(19)$ \\
$+/-$ & $137(9)$ & $103(10)$ \\
$-/+$ & $247(17)$ & $140(14)$ \\
$+/+$ & $753(50)$ & $555(56)$ \\
Missing & 140 & 36 \\
\hline
\end{tabular}

Conditioning, n (\%)

Chemotherapy-based $\mathrm{MAC}^{4} \quad 1118(68)$

TBI-based MAC

\section{$896(88)$}

125 (12)

Post-grafting immunosuppression, $\mathrm{n}(\%)$

\begin{tabular}{lcc} 
CNI + MTX & $1472(90)$ & $523(51)$ \\
CNI + MMF & $58(4)$ & $193(19)$ \\
CNI alone & $46(3)$ & $218(21)$ \\
Other/missing & $57(3)$ & $87(9)$ \\
\hline
\end{tabular}

ATG, n (\%)

Thymoglobuline

Median (IQR) dose, mg/kg

ATG-Fresenius

$\begin{array}{cc}- & 249(30) \\ - & 5.0(5.0-5.6) \\ - & 569(70) \\ & 30(30-35) \\ - & 203\end{array}$

Median (IQR) dose, $\mathrm{mg} / \mathrm{kg}$

Missing

203

HLA: human leucocyte antigen; MSD: HLA-identical sibling donor; BM: bone marrow; PBSC: peripheral blood stem cells; CI: confidence interval; ATG: anti-thymocyte globulin; AML: acute myeloid leukemia; HSCT: hematopoietic stem cell transplantation; IQR: interquartile ranges; CR: complete remission; CMV: cytomegalovirus; MAC: myeloablative conditioning; TBI: total body irradiation; CNI: calcineurin inhibitor; MTX: methotrexate; MMF: mycophenolate mofetil; ${ }^{1}$ defined as t $(8 ; 21)$, $t(15 ; 17)$, inv or del (16), or acute promyelocytic leukemia, these abnormalities only or combined with others; ${ }^{2}$ defined as all cytogenetics not belonging to the good or high risk (including trisomies); ${ }^{3} \mathrm{defined}$ as $11 \mathrm{q} 23$ abnormalities, complex karyotype, abnormalities of chromosomes 5 and $7 ;{ }^{4}$ defined as use of $\geq 6$ Gy TBI, busulfan $>8 \mathrm{mg} / \mathrm{kg}$, or melphalan $>140 \mathrm{mg} / \mathrm{m}^{2}$. 
adjusting for potential confounding factors by multivariate analyses there was no significant impact of the graft type on relapse (HR=1.0, 95\% CI: 0.8-1.2, $P=0.6$ ). In contrast, a more recent year of transplantation ( $\mathrm{HR}=1.1,95 \% \mathrm{C} 1$ : 1.0-1.2, $P=0.02$ ) was associated with a higher risk of relapse. There was no interaction between the association of graft type and the risk of relapse and year of transplantation (interaction test $P=0.38$ ). Unadjusted comparison of relapse incidence between the two groups per 6 -year periods is shown in the Online Supplementary Table S2. We fur-
A

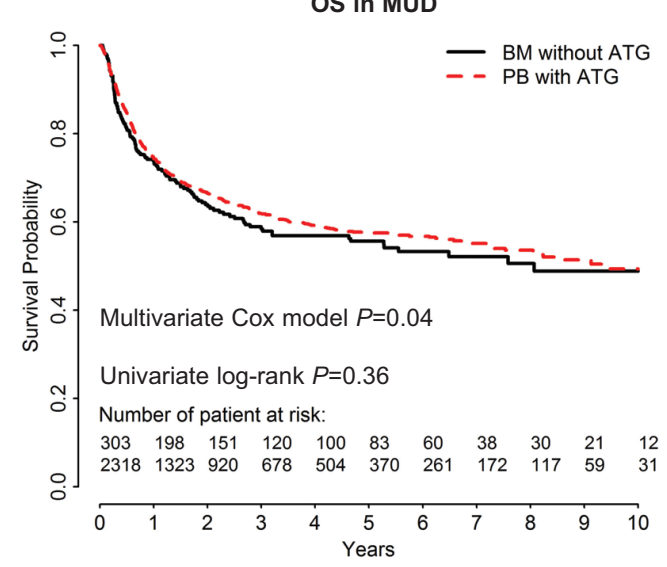

C

RI in MUD

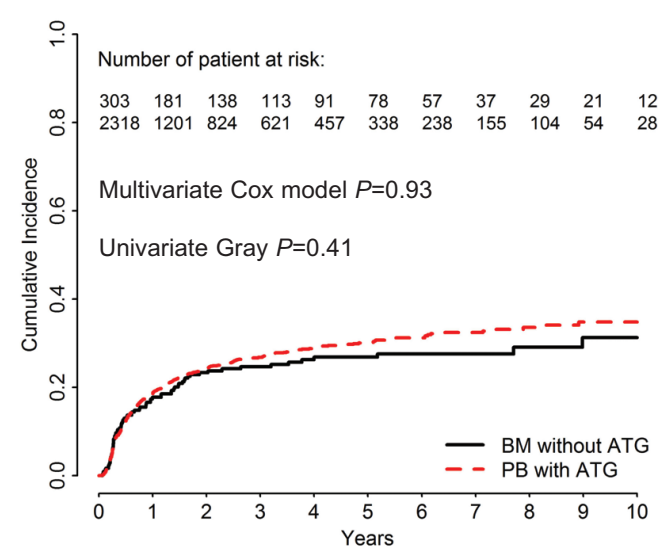

E

CGvHD in MUD

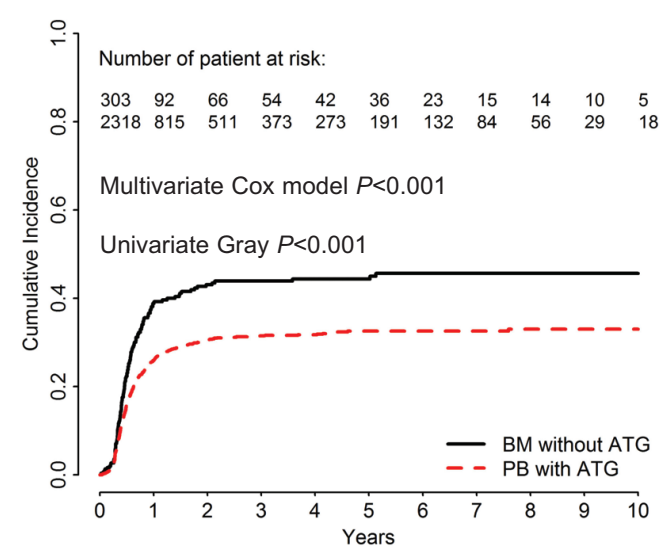

B

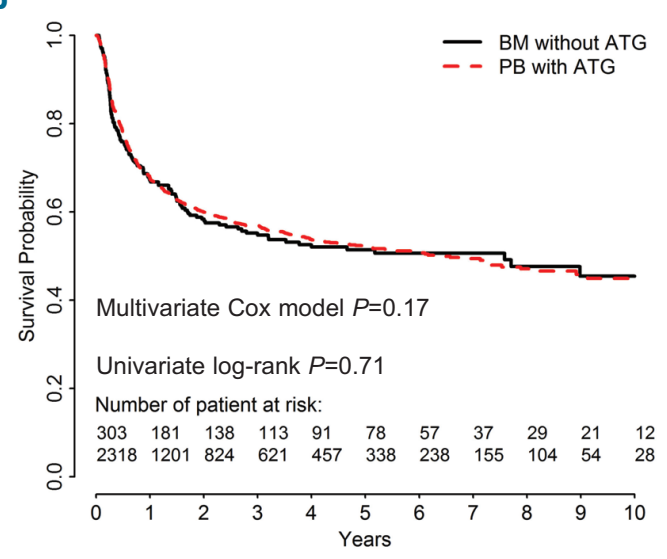

D

NRM in MUD

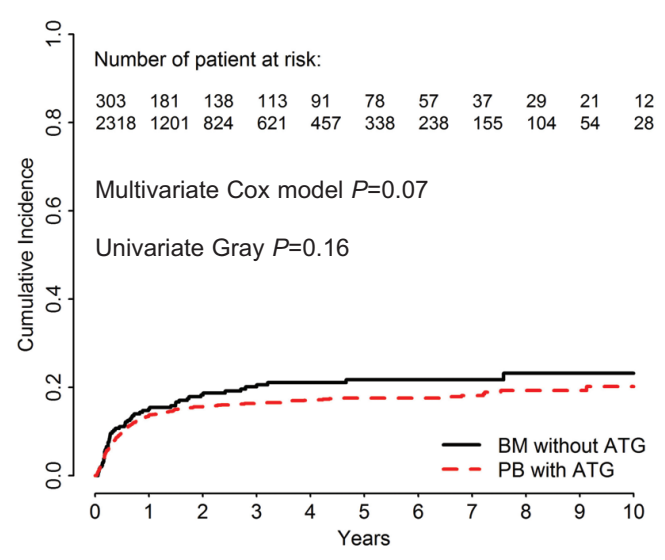

F

GRFS in MUD

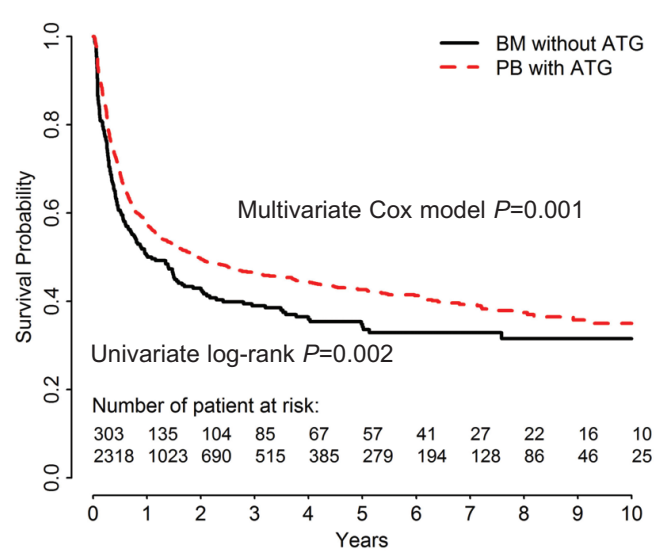

Figure 2. Comparison of outcomes in bone marrow (BM) patients without anti-thymocyte globulin (ATG)and in those given peripheral blood (PB) stem cells with ATG (the dotted line shows the PBSC with ATG curve adjusted for relevant covariates) in the cohort of patients receiving grafts from 10/10 HLA-matched unrelated donors (MUD). (A) Overall survival (OS), $P=0.36$. (B) Leukemia-free survival (LFS), $P=0.71$. (C) Incidence of relapse (RI), $P=0.41$.(D) Non-relapse mortality (NRM), $P=0.16$. (E) Chronic graft-versus-host disease (cGvHD), $P<0.001$. (F) GvHD-free and relapse-free survival (GRFS), $P=0.002$. 
ther investigated whether there was a statistical interaction between the use of TBI in the conditioning regimen and the impact of graft type on relapse risk. Among patients given graft from MSD, there was no such interaction $(P$-value for the interaction test $=0.97)$.

The 2-year CI of NRM was $11.5 \%$ in both groups (Figure 1D). In multivariate analyses, PBSC with ATG was associated with a similar risk of NRM to $B M(H R=1.1$, 95\% CI: 0.8-1.4, $\mathrm{P}=0.7)$. In contrast, older age at transplantation $(\mathrm{HR}=1.5,95 \% \mathrm{CI}: 1.4-1.7, P<0.001)$ and secondary versus de novo $\mathrm{AML}(\mathrm{HR}=1.5,95 \% \mathrm{CI}$ : 1.0-2.1, $P=0.03)$ were associated with a higher NRM while a more recent year of transplantation was associated with a lower NRM (HR=0.7, 95\% C1: 0.6-0.8, $P<0.001)$.

\section{OS and LFS}

The 2-year OS and LFS were 70\% (95\% CI: 68-73\%) and $64 \%(95 \%$ CI: $62-66 \%)$ in BMT without ATG patients versus $68 \%$ (95\% CI: $64-71 \%, \mathrm{P}=0.1)$ and $61 \%$ (95\% CI: $58-64 \%, P=0.06$ ) in PBSC with ATG patients, respectively (Figure 1A-B). After adjusting for potential confounding factors by multivariate analyses, the use of PBSC with ATG was no different to BM with respect to OS (HR=1.0, 95\% CI: 0.8-1.2, $P=0.8)$ and LFS (HR=1.0, 95\% CI: $0.8-1.2, P=0.9)$. In contrast, second CR at transplantation ( $\mathrm{HR}=1.3,95 \% \mathrm{CI}: 1.0-1.5, P=0.03)$, and older patient age $(\mathrm{HR}=1.2,95 \% \mathrm{CI}: 1.1-1.3, P<0.001)$ were associated with worse OS. The same factors were also significantly associated with worse LFS: second CR at transplantation ( $\mathrm{HR}=1.2,95 \% \mathrm{CI}: 1.0-1.5 ; P=0.04)$, and older patient age $(\mathrm{HR}=1.2,95 \% \mathrm{CI}: 1.1-1.2, P<0.001)$.

\section{GRFS}

At 2 years, GRFS was 50\% (95\% CI: $48-53 \%)$ in BMT without ATG patients versus $52 \%$ (95\% CI: $48-55 \%)$ in the PBSC with ATG group $(P=0.8)$ (Figure $1 \mathrm{~F})$. This was confirmed on multivariate analysis including data from all patients (HR=0.9, 95\% CI: 0.8-1.1, $P=0.5)$ as well as in further sensitivity analysis restricted to patients given a combination of a calcineurin inhibitor and methotrexate as GvHD prophylaxis ( $\mathrm{HR}=1.0,95 \% \mathrm{CI}: 0.8-1.2, P=0.9)$. In contrast, female donor to male recipient $(\mathrm{HR}=1.2,95 \% \mathrm{CI}$ : 1.0-1.3, $P=0.01)$, and older patient age $(\mathrm{HR}=1.1,95 \% \mathrm{CI}$ : 1.1-1.1, $P<0.001$ ) were associated with a worse GRFS.

\section{BM without ATG versus PBSC with ATG in MUD recipients \\ Patients}

A total of 2,621 recipients met the inclusion/exclusion criteria. Comprising 303 patients in the BM group and 2,318 in the PBSC with ATG group (Table 3). In comparison to BM recipients, PBSC with ATG patients were older (median 48 years vs. 42 years old), were transplanted more recently (median year of transplantation 2013 versus 2012) and were more frequently transplanted in the first CR (79\% vs. $71 \%)$.

\section{GvHD}

The 100-day incidence of grade II-IV aGvHD was 44\% in BMT without ATG recipients vs. $24 \%$ in PBSC with ATG recipients $(P<0.0001)$. For grade $3-4$, the figures were $17 \%$ (95\% CI: $13-21 \%)$ and 7\% (95\% CI: 6-8\%), respectively $(P<0.001)$. In multivariate analysis, PBSC with ATG patients had a significantly lower incidence of grade II-IV aGvHD than $\mathrm{BM}$ recipients ( $\mathrm{HR}=0.5,95 \%$ CI: 0.4-0.7;
$P<0.001)$. No other factor was significantly associated with grade II-IV aGvHD in multivariate analysis (Online Supplementary Table S4).

The 2-year CI of cGvHD was 43\% (95\% CI: $37-49 \%$ ) in BM without ATG recipients versus $30 \%$ (95\% CI: $28-32 \%)$ in PBSC with ATG patients $(P<0.0001)$ (Figure $2 \mathrm{E})$. On multivariate analysis, PBSC with ATG, in comparison to BM without ATG, was associated with a lower incidence of cGvHD (HR=0.6, 95\% CI: 0.5-0.8, $P=0.0006$ ) (Table 2), but no other factor was significantly associated with cGvHD.

\section{Relapse and non-relapse mortality}

The 2-year CI of relapse was 23\% (95\% CI: 19-29\%) in BMT without ATG recipients versus $24 \%$ (95\% CI: $23-$ $26 \%)$ in PBSC with ATG patients $(P=0.4)$ (Figure 2C). On multivariate analysis, as observed in the MSD cohort, there was no significant impact of the graft type on relapse ( $\mathrm{HR}=1.0,95 \% \mathrm{CI}: 0.8-1.3, P=0.9)$, but a more recent transplantation was significantly associated with a higher risk of relapse ( $\mathrm{HR}=1.2$, 95\% CI: 1.0-1.4, $P=0.03$ ) (Online Supplementary Table S1). There was no interaction between the association of graft type and the risk of relapse and year of transplantation (interaction test $P=0.13$ ). Unadjusted comparison of relapse incidence between the two groups per 6-year periods is shown in the Online Supplementalry Table S2. We further investigated whether there was a statistical interaction between the use of TBI in the conditioning regimen and the impact of the graft type on the relapse risk. Among patients given graft from MUD, there was such an interaction ( $P$-value for the interaction test $=0.05)$. Specifically, in the subgroup of patients given TBI-based

Table 2. Outcomes (multivariate Cox models).

$\begin{array}{cc}\text { iOutcomes } & \text { HR (95\% CI) PBSC } \\ \text { with ATG vs BMT } & P \\ \text { without ATG } & \text { value }{ }^{1}\end{array}$

MSD

\begin{tabular}{lcc} 
Grade II-IV acute GvHD & $0.82(0.64-1.05)$ & 0.12 \\
Chronic GvHD & $0.74(0.59-0.93)$ & 0.01 \\
Relapse incidence & $0.96(0.79-1.16)$ & 0.64 \\
Non relapse mortality & $1.07(0.79-1.44)$ & 0.68 \\
Leukemia-free survival & $0.99(0.84-1.16)$ & 0.89 \\
Overall survival & $0.97(0.81-1.16)$ & 0.75 \\
GvHD-free and relapse-free survival & $0.94(0.81-1.1)$ & 0.45 \\
MUD & & \\
Grade 2-4 acute GvHD & $0.54(0.41-0.71)$ & $<0.001$ \\
Chronic GvHD & $0.62(0.47-0.81)$ & $<0.001$ \\
Relapse incidence & $0.99(0.77-1.27)$ & 0.93 \\
Non relapse mortality & $0.72(0.50-1.02)$ & 0.07 \\
Leukemia-free survival $^{\text {Overall survival }}$ & $0.87(0.72-1.06)$ & 0.17 \\
GvHD-free and relapse-free survival $^{2}$ & $0.80(0.65-0.99)$ & 0.04 \\
\hline
\end{tabular}

HLA: human leucocyte antigen; MSD: HLA-identical sibling donor; MUD: 10/10 HLAmatched unrelated donors; HR: hazard ratio; $\mathrm{CI}$ : confidence interval; BM: bone marrow; BMT: bone marrow transplant; PBSC: peripheral blood stem cells; ATG: anti-thymocyte globulin; GvHD: graft-versus-host disease. ${ }^{1}$ Factors included in the models are: disease status at transplantation, female donor to male recipient or not, cytomegalovirus (CMV) serostatus, use or not of total body irradiation (TBI), year of transplantation, age at transplantation, primary versus secondary acute myeloid leukemia (AML), cytogenetic risk and frailty center effect; ${ }^{2}$ defined as no death, no relapse, no grade III-IV acute GvHD and no extensive chronic GvHD. 
conditioning we observed a lower risk of relapse in PBSC with ATG patients than in BMT without ATG patients ( $\mathrm{HR}=0.56,95 \%$ CI: 0.39-0.81, $P=0.002$ ) while this was not the case in patients given chemotherapy-based conditioning ( $\mathrm{HR}=0.94,95 \% \mathrm{CI}: 0.70-1.26, P=0.68)$.
The 2-year CI of non-relapse mortality was $18 \%$ in BMT without ATG patients versus $16 \%$ in PBSC with ATG patients $(P=0.16)$ (Figure 2D). On multivariate analysis, there was a non-significant trend for lower NRM in PBSC with ATG patients (HR $=0.7,95 \%$ CI: 0.7 -

Table 3. Patient characteristics among 10/10 human leucocyte antigen-matched unrelated donor recipients.

$\begin{array}{lccc}\text { Variable } & \begin{array}{c}\text { BM without ATG } \\ (\mathbf{n}=\mathbf{3 0 3})\end{array} & \begin{array}{c}\text { PBSC with ATG } \\ (\mathbf{n}=\mathbf{2 3 1 8})\end{array} & \text { P } \\ \text { Follow up, median (95\% Cl) } & 5.0(4.0-5.4) & 2.7(2.5-3.0) & <0.001 \\ \text { Age of patient at HSCT (years), Median (IQR) } & 42(31-50) & 48(37-56) & 0.50 \\ \text { Sex, } \mathrm{n}(\%) & 152(50) & 1210(52) & 1108(48)\end{array}$

Missing

0

Karnofsky, n (\%)

$<80$
$\geq 80$
Missing

Status at transplant, $\mathrm{n}(\%)$

CR1

CR2

Cytogenetic risk

Good $^{1}$

Intermediate ${ }^{2}$

Poor $^{3}$

Missing

AML type

Primary

Secondary

Year of transplant, Median (IQR)

Sex mismatch, n (\%)

Female to male

CMV donor/patient, n (\%)

$-/-$

$+/-$

$-1+$

$+/+$

Missing

Conditioning, $\mathrm{n}(\%)$

Chemotherapy -based MAC ${ }^{4}$

TBI-based MAC

Post-grafting immunosuppression, $\mathrm{n}(\%)$

\begin{tabular}{lr}
$\mathrm{CNI}+\mathrm{MTX}$ & $262(86)$ \\
$\mathrm{CNI}+\mathrm{MMF}$ & $17(6)$ \\
$\mathrm{CNI}$ alone & $8(3)$ \\
Other/missing & $16(5)$ \\
\hline
\end{tabular}

ATG, n (\%)

Thymoglobuline

Median (IQR) dose, mg/kg

ATG-Fresenius

Median (IQR) dose, mg/kg

Missing

14

8

\section{6 (2)}

283 (98)

215 (71)

88 (29)

$108(48)$

0

$46(2)$

$2156(98)$

116

1836 (79)

482 (21)

$\begin{array}{cc}30(14) & 176(14) \\ 137(65) & 793(64) \\ 43(21) & 266(22) \\ 93 & 1083\end{array}$

$\begin{array}{cc}277(91) & 2002(86) \\ 26(9) & 316(14)\end{array}$

2012 (2009-2014)

2013 (2011-2015)

0,99

$28(9)$

279 (12)

0.15

99 (34)

608 (27)

27 (9)

84 (28)

85 (29)

179 (8)

703 (32)

741 (33)

87

189 (62)

$$
114 \text { (38) }
$$

1873 (81)

445 (19)

$\begin{array}{cc}262(86) & 1468(63) \\ 17(6) & 556(24) \\ 8(3) & 134(6) \\ 16(5) & 160(7)\end{array}$

$\begin{array}{cc} & 736(42) \\ - & 5.5(5.0-6.0) \\ - & 1013(58) \\ & 40(30-60) \\ - & 569\end{array}$

HLA: human leucocyte antigen; MUD: 10/10 HLA-matched unrelated donors; CI: confidence interval; BM: bone marrow; PBSC: peripheral blood stem cells; AML: acute myeloid leukemia; ATG: anti-thymocyte globulin; HSCT: hematopoietic stem cell transplantation; IQR: interquartile range; CR: complete remission; CMV: cytomegalovirus; MAC: myeloablative conditioning; TBI: total body irradiation; CNI: calcineurin inhibitor; MTX: methotrexate; MMF: mycophenolate mofetil;'defined as t(8;21),t(15;17), inv or del (16), or acute promyelocyticleukemia, these abnormalities only or combined with others; ${ }^{2}$ defined as all cytogenetics not belonging to the good or high risk (including trisomies); ${ }^{3}$ defined as 11q23 abnormalities, complex karyotype, abnormalities of chromosomes 5 and 7 , as well as 3q26 and 17p abnormalities, as defined previously16; ${ }^{4}$ defined as use of $\geq 6$ Gy TBI, busulfan $>8 \mathrm{mg} / \mathrm{kg}$, or melphalan $>140 \mathrm{mg} / \mathrm{m}^{2}$. 
$1.0, P=0.07)$. Factors associated with NRM on multivariate analysis included older age at transplantation $(\mathrm{HR}=1.4,95 \% \mathrm{CI}: 1.3-1.5, P<0.001)$ and secondary $\mathrm{AML}$ ( $\mathrm{HR}=1.4,95 \% \mathrm{CI}: 1.1-1.9, P=0.01)$ while a more recent transplant year was associated with lower NRM (HR=0.8, 95\% CI: 0.7-1.0, $P=0.03$ ).

\section{OS and LFS}

The 2-year OS and LFS were 64\% (95\% CI: $58-70 \%$ ) and $58 \%$ (95\% CI: $53-64 \%)$ in BMT without ATG patients versus $67 \%$ (95\% CI: $64-69 \%, P=0.3)$ and $60 \%$ (95\% CI: $58-62 \%, P=0.7$ ) in PBSC with ATG patients, respectively (Figure $2 \mathrm{~A}-\mathrm{B}$ ). After adjusting for potential confounding factors, the use of PBSC with ATG was associated with a better $\mathrm{OS}(\mathrm{HR}=0.8,95 \% \mathrm{CI}$ : 0.6-1.0, $P=0.04)$ than with $\mathrm{BM}$, but there was no significant difference in LFS between the groups (HR=0.9, 95\% CI: 0.7 $1.1, P=0.2)$. As well as being in the BMT group, older age was significantly associated with a poorer $\mathrm{OS}(\mathrm{HR}=1.2$, 95\% CI: 1.1-1.3, $P<0.0001)$ and a worse LFS $(\mathrm{HR}=1.1$, 95\% CI: 1.1-1.2, $P<0.0001)$.

Causes of death were comparable in both groups expect for a higher frequency of infectious-related death in the PBSC with ATG group (23\% vs. 11\%) (Online Supplementary Table S3).

\section{GRFS}

The 2-year GRFS was 43\% (95\% CI: 37-49\%) versus $50 \%$ (95\% CI: $47-52 \%$ ) in BMT without ATG and PBSC with ATG recipients, respectively $(P=0.002)$ (Figure $2 \mathrm{~F}$ ). This was confirmed in multivariate analysis including data from all patients $(\mathrm{HR}=0.8,95 \%$ CI: 0.6-0.9, $P=0.001)$ as well as in further sensitivity analysis restricted to patients given a combination of a calcineurin inhibitor and methotrexate as GvHD prophylaxis (HR=0.8, 95\% CI: 0.6-0.9, $P=0.01)$. Finally, older patient age was also associated with worse GRFS (HR=1.1, 95\% CI: $1.0-1.1, P=0.004)$ in the whole cohort of patients.

\section{Discussion}

Several studies have now established that the use of PBSC (without ATG) instead of BM is associated with a high incidence of (severe) cGvHD affecting the longterm well-being of the patients. ${ }^{2,5}$ In contrast, several phase III trials have demonstrated a beneficial impact of ATG on cGvHD. ${ }^{12}$ Here, we compare the outcomes of AML patients given BMT without ATG versus PBSC with ATG, in the setting of AML in first or second CR. We elected to restrict the analyses of patients given grafts after myeloablative conditioning since it was a inclusion criteria in most phase III ATG studies, although many AML patients are nowadays transplanted following reduced-toxicity regimens. ${ }^{20,21}$ Several observations can be made.

First, the use of PBSC with ATG was associated with a lower incidence of cGvHD both in the MSD and MUD datasets. This clearly demonstrates that ATG administration is able to counterbalance the high risk of cGvHD associated with the use of PBSC (instead of BMT without ATG).
Importantly, the incidence of relapse was not significantly different in BMT without ATG and in PBSC with ATG patients in both datasets. This may appear surprising given the tight association between cGvHD and graft-versus-leukemia effects, and is consistent with most phase III ATG trials showing, ${ }^{22-24}$ a reduction of cGvHD without an increase in the relapse risk. ${ }^{12}$ Similar findings were observed in the RIC setting in a large registry study. ${ }^{25}$

Given the data of the Soiffer et al. phase III study showing detrimental effects of ATG in patients given TBI-based conditioning regimen, ${ }^{11}$ we investigated whether, in our study, there was a statistical interaction between graft type and the risk of relapse and the use of TBI in the conditioning regimen. Interestingly, we observed a lower risk of relapse only in the subgroup of patients given TBI-based conditioning suggesting no adverse effects of ATG in patients given TBI-based conditioning. These data are in concordance with recent observations reported by our group. ${ }^{26}$

The main endpoint of our retrospective study was GRFS, a relatively new composite endpoint which aims at capturing the rate of cure without ongoing transplantrelated morbidity. ${ }^{27}$ We observed a non-significant difference in GRFS with the two strategies in the cohort of patients given grafts from MSD, and a significantly better GRFS with PBSC with ATG in the cohort of patients given grafts from MUD. We thus advocate that our data might support the use of PBSC with ATG rather than BMT without ATG, in AML patients in CR at transplantation.

An interesting finding of our study was an increasing relapse incidence (but decreasing non relapse mortality incidence) with more recent transplantations. This might be due to a higher proportion at high risk of relapse (as example more patient with persistent minimal residual disease at transplantation) in more recent years of transplantation. Unfortunately, we do not have data on the MRD status at transplantation for many patients included in this survey.

There are some limitations of the study such as its design (it is however unlikely that a prospective randomized phase III trial will address this question in the near future), the lack of data on mutational AML landscape and minimal residual disease, a high proportion of missing cytogenetic data, the lack of ATG dose data for several patients, and some imbalances between the groups. However these imbalances were adjusted for in the multivariate Cox models. The strengths of the study are the number of patients in each group and their relative uniformity (one single disease, all patients in first or second $\mathrm{CR}$ at transplantation, no HLA-mismatches, only myeloablative conditioning regimen).

In conclusion, our data suggest that PBSC transplantation with ATG results in comparable (in case of MSD) or significantly better (in case of MUD) GRFS than BMT without ATG in patients with AML in CR receiving grafts after myeloablative conditioning. These data might support the use of PBSC with ATG compared to BMT without ATG in patients receiving grafts from MSD or MUD after myeloablative conditioning as treatment for AML in CR. 


\section{References}

1. Blaise D, Kuentz M, Fortanier C, et al. Randomized trial of bone marrow versus lenograstim-primed blood cell allogeneic transplantation in patients with early-stage leukemia: a report from the SociÇtÇ Franáaise de Greffe de Moelle. J Clin Oncol. 2000;18(3):537-571.

2. Flowers MED, Parker PM, Johnston LJ, et al. Comparison of chronic graft-versus-host disease after transplantation of peripheral blood stem cells versus bone marrow in allogeneic recipients: long-term follow-up of a randomized trial. Blood. 2002; 100(2):415-419.

3. Stem Cell Trialists' Collaborative G. Allogeneic peripheral blood stem-cell compared with bone marrow transplantation in the management of hematologic malignancies: an individual patient data meta-analysis of nine randomized trials. J Clin Oncol. 2005;23(22):5074-5087.

4. Anasetti C, Logan BR, Lee SJ, et al. Peripheral-blood stem cells versus bone marrow from unrelated donors. $N$ Engl J Med. 2012;367(16):1487-1496

5. Lee SJ, Logan B, Westervelt $\mathrm{P}$, et al. Comparison of patient-reported outcomes in 5-Year survivors who received bone marrow vs peripheral blood unrelated donor transplantation: long-term follow-up of a randomized clinical trial. JAMA Oncol. 2016;2(12):1583-1589

6. Battipaglia G, Ruggeri A, Labopin M, et al. Refined graft-versus-host disease/relapsefree survival in transplant from HLA-identical related or unrelated donors in acute myeloid leukemia. Bone Marrow Transplant. 2018;53(10):1295-1303.

7. Kroger N, Solano C, Wolschke C, et al. Antilymphocyte globulin for prevention of chronic graft-versus-host disease. N Engl J Med. 2016;374(1):43-53.

8. Bacigalupo A, Lamparelli T, Barisione G, et al. Thymoglobulin prevents chronic graftversus-host disease, chronic lung dysfunction, and late transplant-related mortality: long-term follow-up of a randomized trial in patients undergoing unrelated donor transplantation. Biol Blood Marrow Transplant. 2006;12(5):560-565.

9. Walker I, Schultz KR, Toze CL, et al. Thymoglobulin decreases the need for immunosuppression at 12 months after myeloablative and nonmyeloablative unrelated donor transplantation: CBMTG 0801 , a randomized, controlled trial. Blood. 2014; 124(21):38

10. Finke J, Schmoor C, Bethge WA, et al. Long-term outcomes after standard graft- versus-host disease prophylaxis with or without anti-human-T-lymphocyte immunoglobulin in haemopoietic cell transplantation from matched unrelated donors: final results of a randomised controlled trial. Lancet Haematol. 2017; 4(6):e293-e301.

11. Soiffer RJ, Kim HT, McGuirk J, et al Prospective, randomized, double-blind Phase III clinical trial of anti-T-lymphocyte globulin to assess impact on chronic graftversus-host disease-free survival in patients undergoing HLA-matched unrelated myeloablative hematopoietic cell transplantation. J Clin Oncol. 2017;35(36):4003-4011.

12. Baron F, Mohty M, Blaise D, et al. Anti-thymocyte globulin as graft-versus-host disease prevention in the setting of allogeneic peripheral blood stem cell transplantation: a review from the Acute Leukemia Working Party of the European Society for Blood and Marrow Transplantation. Haematologica. 2017;102(2):224-234.

13. Rubio MT, D'Aveni-Piney M, Labopin M, et al. Impact of in vivo T cell depletion in HLA-identical allogeneic stem cell transplantation for acute myeloid leukemia in first complete remission conditioned with a fludarabine iv-busulfan myeloablative regimen: a report from the EBMT Acute Leukemia Working Party. J Hematol Oncol. 2017;10(1):31.

14. Baron F, Ruggeri A, Beohou E, et al. Occurrence of graft-versus-host disease increases mortality after umbilical cord blood transplantation for acute myeloid leukemia: a report from Eurocord and the ALWP of the EBMT. J Intern Med. 2018; 283(2):178-189

15. Grimwade D, Hills RK, Moorman AV, et al. Refinement of cytogenetic classification in acute myeloid leukemia: determination of prognostic significance of rare recurring chromosomal abnormalities among 5876 younger adult patients treated in the United Kingdom Medical Research Council trials. Blood. 2010;116(3):354-365.

16. Baron F, Ruggeri A, Beohou E, et al. RIC versus MAC UCBT in adults with AML: A report from Eurocord, the ALWP and the CTIWP of the EBMT. Oncotarget. 2016; 7(28):43027-43038

17. Ruggeri A, Labopin M, Ciceri F, Mohty M, Nagler A. Definition of GvHD-free relapse-free survival for registry-based studies: an ALWP-EBMT analysis on patients with AML in remission. Bone Marrow Transplant. 2016;51(4):610-611.

18. Andersen PK, Klein JP, Zhang MJ. Testing for centre effects in multi-centre survival studies: a Monte Carlo comparison of fixed and random effects tests. Stat Med. 1999;
18(12):1489-1500

19. Baron F, Labopin M, Ruggeri A, et al Impact of donor type in patients with AML given allogeneic hematopoietic cell transplantation after low-dose TBI-based regimen. Clin Cancer Res. 2018; 24(12):2794 2803.

20. Potdar RR, Gupta S, Giebel S, et al. Current status and Perspectives of irradiation-based conditioning regimens for patients with acute leukemia undergoing hematopoietic stem cell transplantation. Clin Hematol International. 2019;1(1):19-27.

21. Baron F, Labopin M, Peniket A, et al Reduced-intensity conditioning with fludarabine and busulfan versus fludarabine and melphalan for patients with acute myeloid leukemia: A report from the Acute Leukemia Working Party of the European Group for Blood and Marrow Transplantation. Cancer. 2015;121(7):10481055.

22. Weiden PL, Sullivan KM, Flournoy N, Storb $\mathrm{R}$, Thomas ED, and the Seattle Marrow Transplant T. Antileukemic effect of chronic graft-versus-host disease. Contribution to improved survival after allogeneic marrow transplantation. N Engl J Med. 1981; 304(25):1529-1533

23. Baron F, Maris MB, Sandmaier BM, et al Graft-versus-tumor effects after allogeneic hematopoietic cell transplantation with nonmyeloablative conditioning. J Clin Oncol. 2005;23(9):1993-2003.

24. Baron F, Labopin M, Niederwieser D, et al Impact of graft-versus-host disease after reduced-intensity conditioning allogeneic stem cell transplantation for acute myeloid leukemia: a report from the Acute Leukemia Working Party of the European group for blood and marrow transplantation. Leukemia. 2012;26(12):2462-2468.

25. Baron F, Labopin M, Blaise D, et al. Impact of in vivo T-cell depletion on outcome of AML patients in first CR given peripheral blood stem cells and reduced-intensity conditioning allo-SCT from a HLA-identical sibling donor: a report from the Acute Leukemia Working Party of the European Group for Blood and Marrow Transplantation. Bone Marrow Transplant. 2014;49(3):389-396

26. Nagler A, Labopin M, Dholaria B, et al Impact of antithymocyte globulin on outcomes of allogeneic hematopoietic cell transplantation with TBI. Blood Adv. 2019; 3(13):1950-1960.

27. Holtan SG, DeFor TE, Lazaryan A, et al. Composite end point of graft-versus-host disease-free, relapse-free survival after allogeneic hematopoietic cell transplantation. Blood. 2015;125(8):1333-1338. 